\title{
Bone healing of distal radius nonunion treated with bridge plating with bone graft substitutes in combination with systemic romosozumab administration: A case report
}

\author{
Takuya Uemura, MD, $\mathrm{PhD}^{1}{ }^{10}$, Koichi Yano, MD, $\mathrm{PhD}^{2}$, Kiyohito Takamatsu, MD, $\mathrm{PhD}^{3}$, \\ Yusuke Miyashima, MD' ${ }^{1}$, Hiroyuki Yasuda, MD, PhD ${ }^{1}$, Sadahiko Konishi, MD, $\mathrm{PhD}^{1}{ }^{\mathbb{D}}$, \\ Hiroaki Nakamura, MD, $\mathrm{PhD}^{4}$ D \\ 'Department of Orthopaedic Surgery, Osaka General Hospital of West Japan Railway Company, Osaka, Japan \\ ${ }^{2}$ Department of Orthopaedic Surgery, Seikeikai Hospital, Osaka, Japan \\ ${ }^{3}$ Department of Orthopaedic Surgery, Yodogawa Christian Hospital, Osaka, Japan \\ ${ }^{4}$ Department of Orthopaedic Surgery, Osaka City University Graduate School of Medicine, Osaka, Japan
}

Romosozumab is a humanized, anti-sclerostin monoclonal, bone-forming antibody with a dual effect which increases bone formation and decreases bone resorption and is indicated to treat osteoporosis. ${ }^{[1-6]}$ Initial animal and preclinical researches have revealed that romosozumab enhances fracture healing, and romosozumab would be expected to accelerate bone healing of delayed union or nonunion as a pharmacological systemic treatment. ${ }^{[4-6]}$ However, there have been no clinical reports about romosozumab therapy for the nonunion of long bones,

Received: March 02, 2021

Accepted: March 20, 2021

Published online: June 11, 2021

Correspondence: Takuya Uemura, MD, PhD. Department of Orthopaedic Surgery, Osaka General Hospital of West Japan Railway Company, 1-2-22 Matsuzakicho, Abeno-ku, Osaka 545-0053, Japan.

E-mail: t-uemura@med.osaka-cu.ac.jp

Doi: $10.52312 / j d r s .2021 .82661$

Citation: Uemura T, Yano K, Takamatsu K, Miyashima Y, Yasuda $\mathrm{H}$, Konishi $\mathrm{S}$, et al. Bone healing of distal radius nonunion treated with bridge plating with bone graft substitutes in combination with systemic romosozumab administration: A case report. Jt Dis Relat Surg 2021;32(2):526-530.

(C2021 All right reserved by the Turkish Joint Diseases Foundation

This is an open access article under the terms of the Creative Commons Attribution-NonCommercial License, which permits use, distribution and reproduction in any medium, provided the original work is properly cited and is not used for commercial purposes (http://creativecommons.org/licenses/by-nc/4.0/).

\section{ABSTRACT}

Romosozumab is a humanized, anti-sclerostin monoclonal antibody used to treat osteoporosis, which increases bone formation and decreases bone resorption. It enhances fracture healing and systemic romosozumab administration may have therapeutic potentials for accelerating bone healing of even nonunion. Herein, a 61-year-old heavy smoker male with distal radius nonunion who achieved successful bone union by combination therapy of romosozumab and spanning distraction plate fixation with bone graft substitutes was presented. Through the dorsal approach, atrophic comminuted nonunion of the distal radius was sufficiently debrided. Reduction of the distal radius was performed using indirect ligamentotaxis, and a 14-hole locking plate was fixed from the third metacarpal to the radial shaft. A beta ( $\beta$ ) tricalcium phosphate block was mainly packed into the substantial metaphyseal bone defect with additional bone graft from the resected ulnar head. Postoperatively, systemic administration of monthly romosozumab was continued for six months. Complete bone union was achieved 20 weeks postoperatively and the plate was, then, removed. Wrist extension and flexion improved to $75^{\circ}$ and $55^{\circ}$, respectively, without pain, and grip strength increased 52 weeks postoperatively from $5.5 \mathrm{~kg}$ to $22.4 \mathrm{~kg}$. During romosozumab treatment, bone formation marker levels increased rapidly and finally returned to baseline, and bone resorption marker levels remained low. In conclusion, combination of systemic romosozumab administration and grafting $\beta$-tricalcium phosphate with bridge plating provides an effective treatment option for difficult cases of comminuted distal radius nonunion with risk factors such as smoking, diabetes, and fragility.

Keywords: Delayed union, distal radius fracture, fracture healing, locking plate, osteoporosis, sclerostin.

particularly using bone graft substitutes together. Herein, we present the first case of a heavy smoker with comminuted distal radius nonunion treated successfully with combination therapy of systemic 
romosozumab administration and grafting beta $(\beta)$-tricalcium phosphate with spanning distraction plate fixation.

\section{CASE REPORT}

A 61-year-old, left-hand-dominant man fell down on the street and injured his right wrist. He sustained a distal radius fracture and was initially treated with a cast immobilization at an external center. Subsequently, surgical treatment was recommended, as the fracture gradually displaced in the cast; however, he selected conservative treatment with maintaining the cast. Three months after the initial injury, he was referred to our hand surgery clinic and complained of persistent wrist pain (Visual Analog Scale score: 82 ) and forearm deformity due to distal radius nonunion. He was a smoker with 35 cigarettes per day for over 40 years and had diabetes requiring insulin injection treatment. He also had a history of a left intertrochanteric femoral fracture and untreated osteoporosis. His bone mineral density was extremely low $\left(0.603 \mathrm{~g} / \mathrm{cm}^{2}\right)$, and he had a T-score lower than 2.0 standard deviations on dual energy X-ray absorptiometry. Biochemical markers of bone turnover showed elevated levels of both bone formation markers, including $84.6 \mathrm{ng} / \mathrm{mL}$ of serum procollagen type I N propeptide (P1NP) and $47.5 \mu \mathrm{g} / \mathrm{L}$ of bone-specific alkaline phosphatase (BAP), and bone resorption markers, including $18.0 \mathrm{nmoL} \mathrm{BCE} / \mathrm{L}$ of serum $\mathrm{N}$-terminal telopeptide (NTX) and $496 \mathrm{mU} / \mathrm{dL}$ of serum tartrate-resistant acid phosphatase (TRACP)-5b. On physical examination, wrist extension and flexion were restricted to $50^{\circ}$ and $40^{\circ}$, respectively, with marked pain. Grip strength was $5.5 \mathrm{~kg}$ in the right hand, compared to $25 \mathrm{~kg}$ in the left hand. Radiographs showed atrophic comminuted nonunion and radial deviation of the distal radius with ulnar abutment by $8 \mathrm{~mm}$ (Figure 1). Computed tomography (CT) showed the dorsally displaced intraarticular comminuted distal fragments with metaphyseal bone loss. Magnetic resonance imaging showed an increased signal intensity in the proximal ulnar pole of the lunate due to ulnar impaction and no necrosis of the distal radius fragments.

We decided to perform a dorsal spanning distraction plate fixation with bone graft substitute for the treatment of the distal radius nonunion, since the distal metaphyseal fragments were comminuted and fragile, with severe metaphyseal bone loss and osteopenia. ${ }^{[7,8]} \mathrm{A}$ written informed consent was
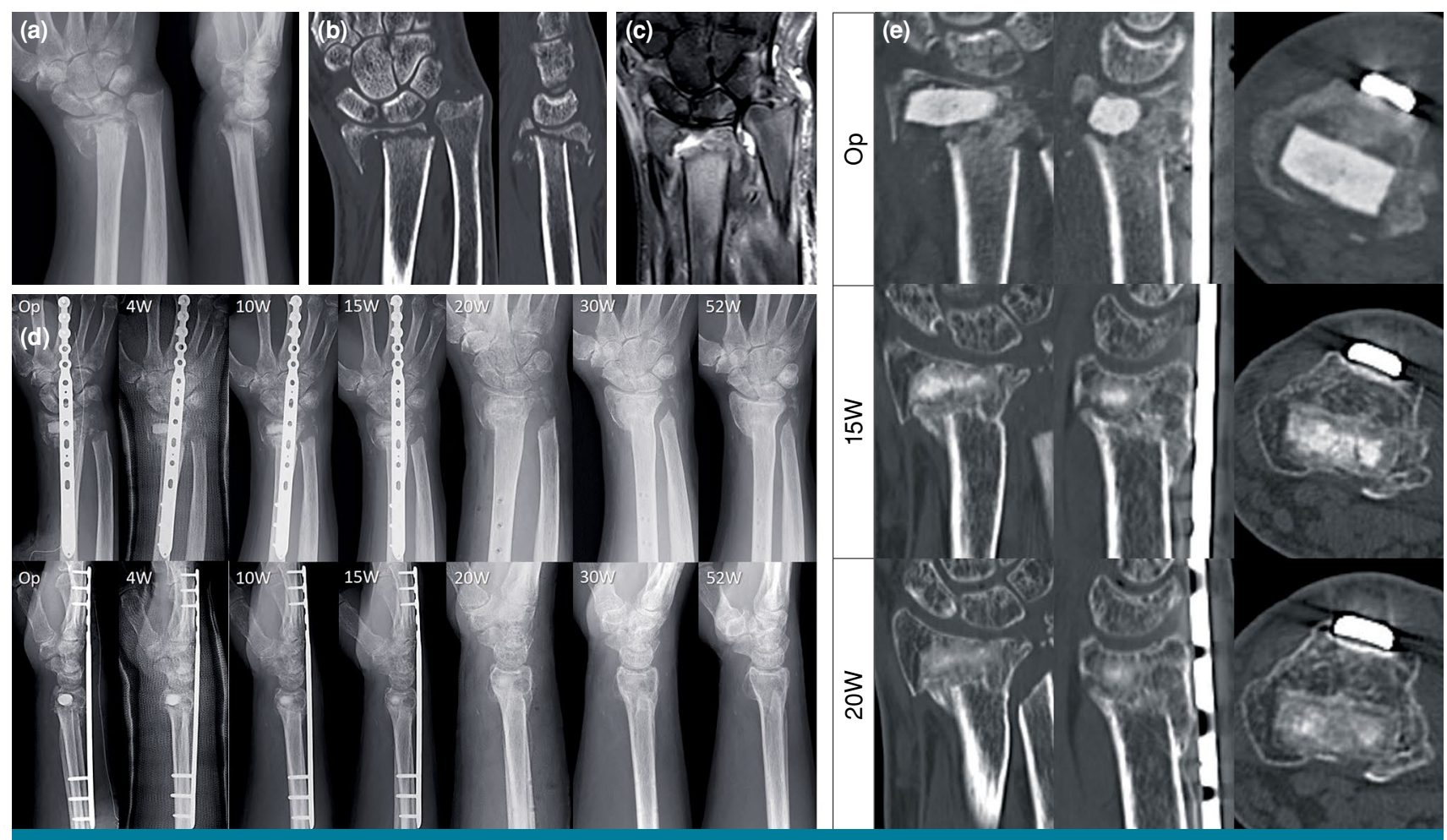

FIGURE 1. (a) Preoperative radiography, (b) CT, and (c) MRI images of right wrist; (d) Intraoperative and postoperative radiographs of right wrist; (e) Postoperative CT images of right wrist.

CT: Computed tomography; MRI: Magnetic resonance imaging. 


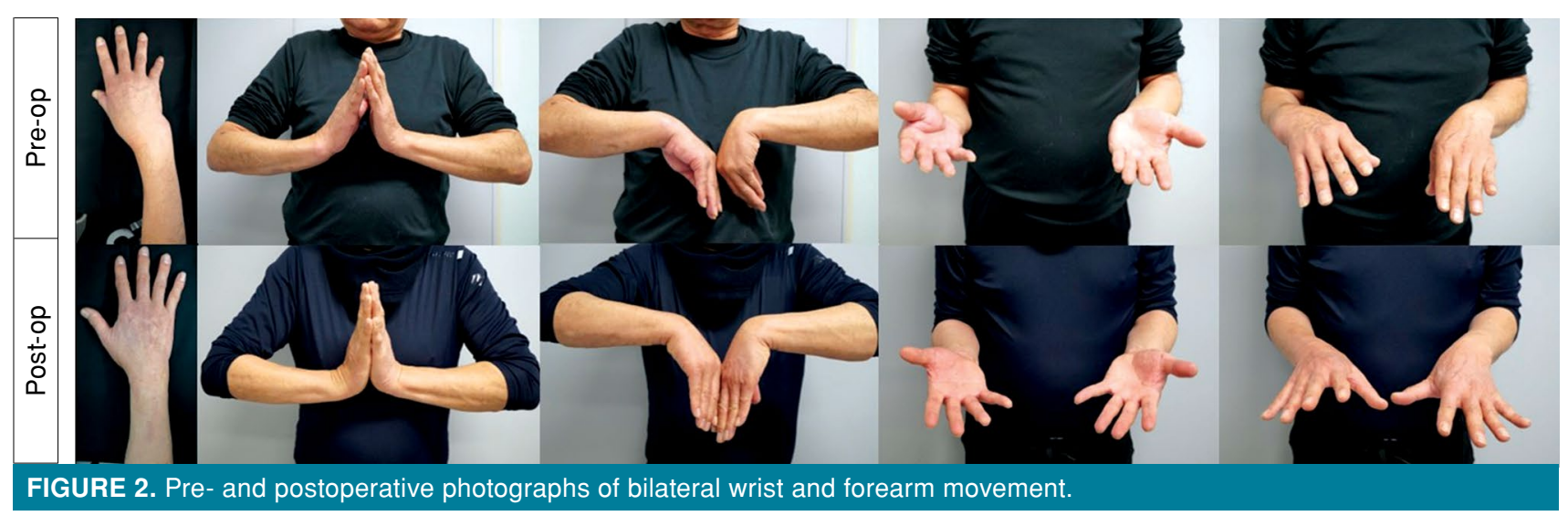

obtained from the patient. Through the first dorsal approach opening the third compartment, the distal radius nonunion was visualized. At first, ulnar head of $15 \mathrm{~mm}$ in length was resected for the treatment of ulnar abutment. Next, the nonunion was sufficiently debrided. Acceptable reduction involving appropriate radial inclination and volar tilt was possible by longitudinal distraction with the use of indirect capsular ligamentotaxis. In addition, a 14-hole straight locking plate of A.L.P.S. ${ }^{\mathrm{TM}}$ Distal Fibula Plating System (Zimmer Biomet, IN, USA) was inserted under the finger extensors from the second incision over the shaft of the third metacarpal to the third incision over the proximal radial shaft, through the first incision of the nonunion site. The plate was fixed with three bicortical locking screws in the third metacarpal and proximal radius shaft, respectively, while keeping and confirming the reduction position under fluoroscopy. Finally, a $\beta$-tricalcium phosphate block (OSferion ${ }^{\circledR}$ A2, Olympus, Tokyo, Japan) was mainly packed into the substantial metaphyseal bone defect with additional cancellous bone chip grafting from the resected ulnar head.

Postoperatively, systemic monthly administration of romosozumab (210 $\mathrm{mg}$, subcutaneous injection, EVENITY ${ }^{\circledR}$, Amgen, CA, USA) was started to promote bone healing and treat osteoporosis and it was continued for six months. Cast immobilization of the wrist was maintained for 10 weeks postoperatively, as partial bone union began to be observed on radiographs 10 weeks after surgery. Complete bone union with consolidation was achieved as evidenced by radiographs and CT images 20 weeks after surgery (Figure 1). Subsequently, the plate was removed and progressive wrist motion was initiated. Fifty-two weeks after surgery, wrist extension and flexion increased to $75^{\circ}$ and $55^{\circ}$, respectively, without wrist pain, and the grip strength increased to $22.4 \mathrm{~kg}$ in the right hand (Figure 2). The patient-reported outcomes, as measured by the Quick Disability of the Arm, Shoulder, and Hand (QuickDASH) score and the Hand20 questionnaires, were improved from 64 and 56 preoperatively to 2.3 and 1.0 postoperatively, respectively. The patient was satisfied with no wrist pain and the appearance of the corrected forearm. At four weeks after romosozumab administration, the levels of serum P1NP and BAP as bone formation markers further increased rapidly to $91.9 \mathrm{ng} / \mathrm{mL}$ and $67.2 \mu \mathrm{g} / \mathrm{L}$, respectively, and the levels of serum NTX and TRACP-5b as bone resorption markers decreased to $17.8 \mathrm{nmoL} \mathrm{BCE} / \mathrm{L}$ and $443 \mathrm{mU} / \mathrm{dL}$, respectively. At six months after romosozumab administration, the levels of serum P1NP and BAP returned to nearly baseline levels of $53.9 \mathrm{ng} / \mathrm{mL}$ and $34.4 \mu \mathrm{g} / \mathrm{L}$, respectively, while the levels of serum NTX and TRACP-5b remained suppressed at $12.5 \mathrm{nmoL}$ BCE/L and $402 \mathrm{mU} / \mathrm{dL}$, respectively.

\section{DISCUSSION}

Sclerostin is a glycoprotein, secreted primarily by osteocytes, which inhibits Wnt signaling and decreases bone formation..$^{[1-3]}$ Romosozumab is an anti-sclerostin monoclonal antibody that increases bone formation and decreases bone resorption..$^{[1-3]}$ In several animal fracture or osteotomy models, systemic administration of romosozumab promoted fracture healing and increased bone strength. ${ }^{[4-6]}$ In contrast, it did not accelerate hip and tibial fracture healing clinically in randomized, placebo-controlled studies. ${ }^{[2,3]}$ However, this can be attributed to the fact that these study populations might not have been at a sufficient risk for delayed healing to demonstrate benefits from the romosozumab intervention for acceleration of fracture healing. Some authors also recommend that studies of at-risk patients for delayed union or nonunion such as smokers and patients 
with diabetes are needed to explore the potential of romosozumab to accelerate fracture healing. In the present case of a heavy smoker and diabetes with distal radius nonunion, romosozumab treatment was effective for bone healing, despite using a bone graft substitute.

In our case, bone formation marker levels rapidly increased, since the onset of romosozumab treatment, and returned to baseline levels six months after the administration. In contrast, bone resorption marker levels were suppressed rapidly and remained below for six months. These changes in the bone turnover markers during romosozumab treatment are very similar to those in a previous randomized, placebo-controlled trial of 7,180 postmenopausal women with osteoporosis treated with romosozumab, indicating that systemic administration of romosozumab exerted its effects by enhancing bone remodeling for the nonunion site. ${ }^{[1]}$

Recently, volar locking plates with or without bone grafting have been used increasingly for the corrective osteotomy treatment of malunited distal radius fractures, alternative to the traditional technique of using dorsal plates with bone grafting. ${ }^{[9-16]}$ However, external fixation or spanning bridge plate fixation is indicated for the difficult cases, such as the present case, in which the distal fragments are too comminuted and fragile to fix with the volar or dorsal locking plates. ${ }^{[7,8]}$ Mithani et al. ${ }^{[7]}$ reported eight cases of distal radius nonunion treated with the dorsal spanning distraction plates. Although it was unclear how many cases the bone graft substitutes were used in, bone union was achieved in all of the cases, and the bridge plate was removed at an average of 148 days. The mean DASH scores improved from 70.5 preoperatively to 27.6 postoperatively. The mean wrist extension and flexion increased postoperatively to $36.4^{\circ}$ and $39.5^{\circ}$, respectively. In the present case, the bridge plate was removed at 140 days, indicating that bone healing time was comparable to the eight cases despite using mainly bone graft substitutes without use of an iliac bone graft. The clinical outcomes involving the postoperative wrist motion and DASH scores were superior to those in the eight cases. We believe that the systemic romosozumab administration contributes to acceleration of bone healing for the nonunion, leading to the better clinical outcomes. Moreover, the use of the bone graft substitutes is feasible and helpful for avoiding troublesome complications associated with autogenous bone grafting such as donor-site morbidity, persistent pain, sensory nerve injury, hematoma, and infection. ${ }^{[7,12,17]}$
In conclusion, although further comparative studies are needed, combination of systemic romosozumab administration and bridge plating with bone graft substitutes provides a useful treatment option for difficult cases of comminuted distal radius nonunion with risk factors such as heavy smoking, diabetes, and fragility.

\section{Declaration of conflicting interests}

The authors declared no conflicts of interest with respect to the authorship and/or publication of this article.

\section{Funding}

The authors received no financial support for the research and/or authorship of this article.

\section{REFERENCES}

1. Cosman F, Crittenden DB, Grauer A. Romosozumab treatment in postmenopausal osteoporosis. N Engl J Med 2017;376:396-7.

2. Schemitsch EH, Miclau T, Karachalios T, Nowak LL, Sancheti P, Poolman RW, et al. A randomized, placebocontrolled study of romosozumab for the treatment of hip fractures. J Bone Joint Surg [Am] 2020;102:693-702.

3. Bhandari M, Schemitsch EH, Karachalios T, Sancheti P, Poolman RW, Caminis J, et al. Romosozumab in skeletally mature adults with a fresh unilateral tibial diaphyseal fracture: A Randomized phase-2 study. J Bone Joint Surg [Am] 2020;102:1416-26.

4. Suen PK, He YX, Chow DH, Huang L, Li C, Ke HZ, et al. Sclerostin monoclonal antibody enhanced bone fracture healing in an open osteotomy model in rats. J Orthop Res 2014;32:997-1005.

5. Cui L, Cheng H, Song C, Li C, Simonet WS, Ke HZ, et al. Time-dependent effects of sclerostin antibody on a mouse fracture healing model. J Musculoskelet Neuronal Interact 2013;13:178-84.

6. Ominsky MS, Li C, Li X, Tan HL, Lee E, Barrero M, et al. Inhibition of sclerostin by monoclonal antibody enhances bone healing and improves bone density and strength of nonfractured bones. J Bone Miner Res 2011;26:1012-21.

7. Mithani SK, Srinivasan RC, Kamal R, Richard MJ, Leversedge FJ, Ruch DS. Salvage of distal radius nonunion with a dorsal spanning distraction plate. J Hand Surg Am 2014;39:981-4.

8. Lauder A, Hanel DP. Spanning bridge plate fixation of distal radial fractures. JBJS Rev 2017;5:01874474201702000-00002.

9. Mugnai R, Tarallo L, Lancellotti E, Zambianchi F, Di Giovine E, Catani F, et al. Corrective osteotomies of the radius: Grafting or not? World J Orthop 2016;7:128-35.

10. Disseldorp DJ, Poeze M, Hannemann PF, Brink PR. Is bone grafting necessary in the treatment of malunited distal radius fractures? J Wrist Surg 2015;4:207-13.

11. Tarallo L, Mugnai R, Adani R, Catani F. Malunited extra-articular distal radius fractures: Corrective osteotomies using volar locking plate. J Orthop Traumatol 2014;15:285-90.

12. Ozer K, Kiliç A, Sabel A, Ipaktchi K. The role of bone allografts in the treatment of angular malunions of the distal radius. J Hand Surg Am 2011;36:1804-9. 
13. Wada T, Tatebe M, Ozasa $Y$, Sato O, Sonoda T, Hirata H, et al. Clinical outcomes of corrective osteotomy for distal radial malunion: a review of opening and closing-wedge techniques. J Bone Joint Surg [Am] 2011;93:1619-26.

14. Miyake J, Murase T, Moritomo H, Sugamoto K, Yoshikawa H. Distal radius osteotomy with volar locking plates based on computer simulation. Clin Orthop Relat Res 2011;469:1766-73.

15. Sato K, Nakamura T, Iwamoto T, Toyama Y, Ikegami H, Takayama S. Corrective osteotomy for volarly malunited distal radius fracture. J Hand Surg Am 2009;34:27-33, 33.e1.

16. Shintani K, Kazuki K, Yoneda M, Uemura T, Okada M, Takamatsu K, et al. Computer-assisted three-dimensional corrective osteotomy for malunited fractures of the distal radius using prefabricated bone graft substitute. J Hand Surg Asian Pac Vol 2018;23:479-86.

17. Westrich GH, Geller DS, O'Malley MJ, Deland JT, Helfet DL. Anterior iliac crest bone graft harvesting using the corticocancellous reamer system. J Orthop Trauma 2001;15:500-6. 